\title{
New option for improving hematological recovery: suppression of luteinizing hormone
}

Harold K. Elias and Marcel R.M. van den Brink

Department of Immunology and Department of Medicine, Memorial Sloan Kettering Cancer Center, New York, NY, USA

E-mail: MARCEL R.M. VAN DEN BRINK - vandenbm@mskcc.org

doi:10.3324/haematol.2020.274969

C urrent treatment modalities in leukemia are limited by bone marrow (BM) toxicity, a common adverse effect of cytotoxic chemotherapy and transplant-related conditioning regimens, resulting in an increased risk of bleeding and infections. Strategies to protect the BM from cytotoxic injury could augment hematopoietic recovery and improve overall patient outcomes.

Hematopoietic recovery following cytotoxic therapies and irradiation is dependent on the maintenance of a rare population of hematopoietic stem cells (HSCs) - which have the ability to sustain long-term hematopoietic recovery. ${ }^{1,2}$ Following HSC transplant, there is evidence of decreased BM cellularity ${ }^{3}$ and diminished colony-forming capacity ${ }^{4-6}$ which could last up to approximately 5 years. Growing evidence attributes these functional defects to several intrinsic and extrinsic regulators which orchestrate radiation-induced senescent and pro-apoptotic programs, thereby dictating HSC fate. ${ }^{7,8}$ Several radioprotective agents have been identified, ${ }^{9}$ but very few mitigate radiation toxicity in the post-injury setting. Historically, mouse studies have informed post-irradiation strategies to promote HSC regeneration which are either cytokinebased, such as a combination of stem cell factor, FMS-like tyrosine kinase 3 ligand, megakaryocyte growth and development factor (MGDF) and Interleukin-3 (IL-3), ${ }^{10}$ single agent IL-33, ${ }^{11}$ or inhibitors targeting PTP ${ }^{12}$ - none of which have been confirmed in the clinical setting. Cognate receptors for sex hormones and luteinizing hormone (LH)-releasing hormone (LHRH) have been identified on HSC and implicated in their function. ${ }^{13-15}$ For example, LH can induce HSC expansion in vitro. ${ }^{13}$ Moreover, preclinical studies targeting the sex-steroid axis, have demonstrated enhanced hematopoietic stem cell function and immune recovery, following sex-steroid ablation $^{16-18}$ and LHRH-antagonism. ${ }^{13}$

In this issue of Haematologica, Dalle and colleagues ${ }^{19}$ provide clinical evidence of BM recovery and long-term hematopoietic reconstitution following targeted therapy of the sex-steroid axis. They conducted a retrospective

\section{Steady-state}

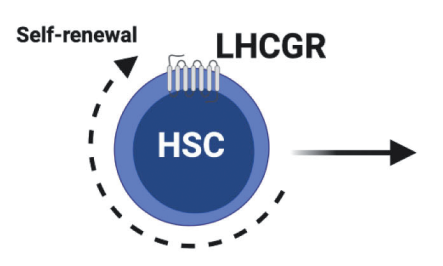

\section{Radiation chemotherapy}

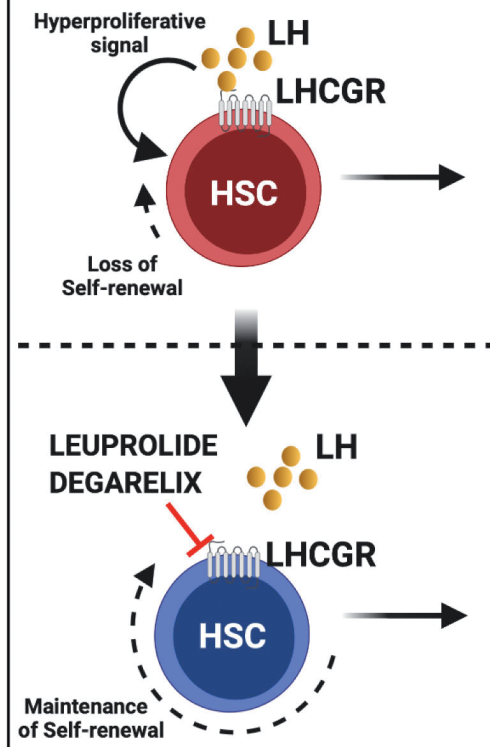

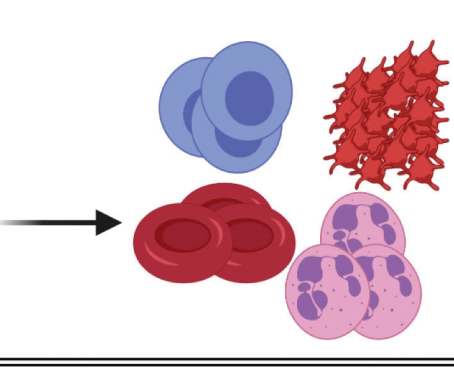

HS HStu,PC

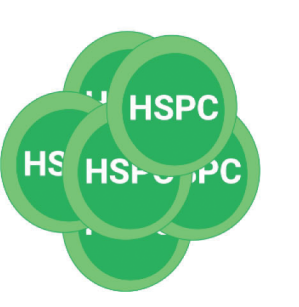

\begin{abstract}
(2)
\end{abstract}
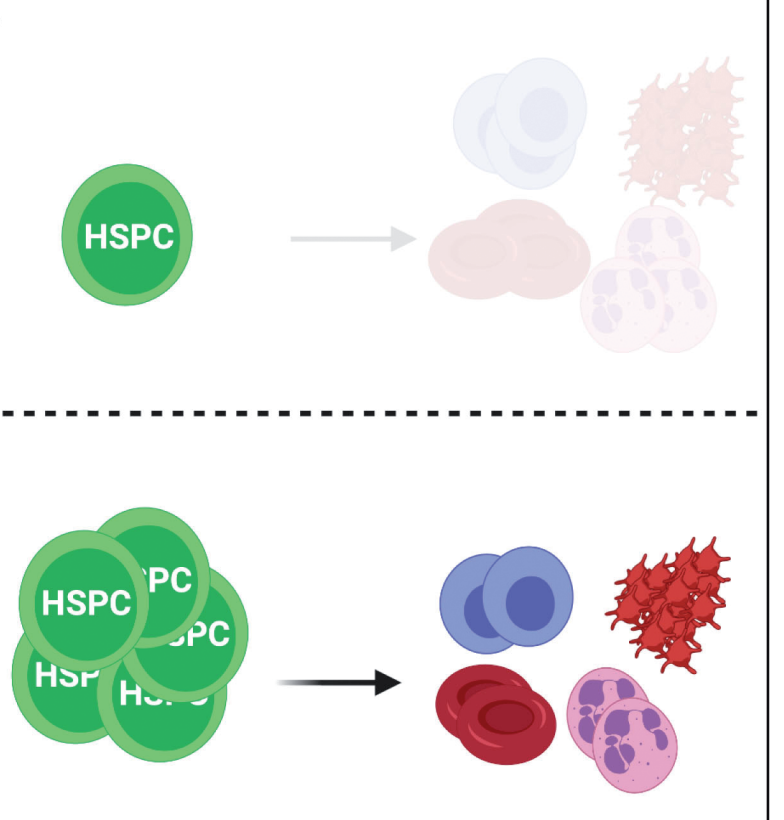

Figure 1. Schematic model of luteinizing hormone-releasing hormone antagonism mediated cytoprotection which promotes hematopoietic stem cell recovery following haematopoietic injury. Dalle et al. ${ }^{19}$ provide clinical evidence for bone marrow recovery and long-term hematopoietic reconstitution with luteinizing hormone-releasing hormone (LHRH) antagonism (leuprolide) in leukemia patients following chemotherapy. HSC: hematopoietic stem cell; HSPC: hematopoietic stem and progenitor cell; LHCGR: luteinizing hormone/choriogonadotropin receptor; LH: luteinizing. hormone. Figure created with BioRender.com. 
study of premenopausal women with leukemia treated with intensive chemotherapy and investigated the impact of leuprolide (gonadotropin-releasing hormone analogue) on long-term hematopoietic reconstituting ability. Their findings established an association between leuprolide use in leukemic patients and sustained recovery in blood counts. Additionally, patients with acute myeloid leukemia treated with leuprolide showed higher longterm hemoglobin levels and fewer blood transfusions. Notably, leuprolide treatment had no impact on either overall or event free survival. Finally, multivariate analysis confirmed that leuprolide administration showed an independent association with long-term hematological recovery.

This retrospective clinical study seeks to build upon previous work showing that sex steroid ablation and abrogation of LH can have beneficial effects on hematopoietic reconstitution in preclinical mouse models. However, the study raises several unanswered questions. Firstly, what would be an ideal clinical window and dosage for leuprolide administration following chemotherapy and whether that impacts association with recovery? The preclinical studies with LHRH-antagonists were protective when administered within 24 hours after radiation. ${ }^{13}$ The current study was limited by sample size to determine statistical significance. Secondly, in relapse cases, where reinduction chemotherapy and irradiation is the standard of care, is additional leuprolide required to help boost hematological tolerance, thereby mitigating hematopoietic stress and temporary cytopenias? Thirdly, are the effects of leuprolide on hematopoietic recovery restricted to BM malignancies or could it be repurposed for treatment of other malignant and non-malignant diseases with BM involvement? Finally, from a mechanistic perspective, recent work demonstrating a role for estrogens in regulating HSC proliferation and function ${ }^{14,15}$ begs the question: are these effects specific to $\mathrm{LH}$ or sex steroids? Considering the rationale for leuprolide to protect against chemoradiation induced premature ovarian failure, ${ }^{20,21}$ preserved estrogen levels could explain the indirect beneficial effects of leuprolide on hematopoietic recovery. Hence, this warrants additional clinical studies accounting for ovarian failure, as that interpretation would restrict the potential utility of this therapy to a younger cohort. These findings also suggest a role of HSC extrinsic factors and raise the question whether leuprolide has a similar cytoprotective effect on the BM microenvironment?

In conclusion, the work by Dalle et al. ${ }^{19}$ highlights a potential new therapeutic option for improving hematological recovery in patients undergoing intensive chemotherapy and transplant conditioning regimens, by boosting post-injury long-term hematopoietic reconstitution; although follow-up clinical investigations are warranted for the rational development of leuprolide as a stand-alone therapy, or in conjunction with other agents. This study also underscores the relevance of mouse models to explore additional markers and molecular underpinnings which confer survival advantage in post-irradiated HSC and BM, as those discoveries will direct us to novel non-cellular approaches to promote hematopoietic recovery and serve as effective therapies against BM toxicity.

\section{Disclosures}

MvdB has received research support and stock options from Seres; has received stock options from Notch Therapeutics; has received royalties from Wolters Kluwer; has consulted, received honorarium from or participated in advisory boards for Seres Therapeutics, Jazz Pharmaceuticals, Rheos, Therakos, WindMIL Therapeutics, Amgen, Merck \& Co, Inc., Magenta Therapeutics, Frazier Healthcare Partners, Nektar Therapeutics, Notch Therapeutics, Forty Seven Inc., Priothera, Ceramedix, DKMS, Pharmacyclics (Spouse), Kite Pharmaceuticals (Spouse); has IP Licensing with Seres Therapeutics and Juno Therapeutics and holds a fiduciary role on the Foundation Board of DKMS (a nonprofit organization).

\section{Contributions}

HKE and MRMvdB have contributed equally.

\section{References}

1. Orkin SH, Zon LI. Hematopoiesis: an evolving paradigm for stem cell biology. Cell. 2008;132(4):631-644

2. Bryder D, Rossi DJ, Weissman IL. Hematopoietic stem cells: the paradigmatic tissue-specific stem cell. Am J Pathol. 2006;169(2):338-346.

3. Arnold R, Schmeiser T, Heit W, et al. Hemopoietic reconstitution after bone marrow transplantation. Exp Hematol. 1986;14(4):271-277.

4. del Canizo C, Lopez N, Caballero D, et al. Haematopoietic damage persists 1 year after autologous peripheral blood stem cell transplantation. Bone Marrow Transplant. 1999;23(9):901-905.

5. Domenech J, Linassier C, Gihana E, et al. Prolonged impairment of hematopoiesis after high-dose therapy followed by autologous bone marrow transplantation. Blood. 1995;85(11):3320-3327.

6. Vellenga E, Sizoo W, Hagenbeek A, Lowenberg B. Different repopulation kinetics of erythroid (BFU-E), myeloid (CFU-GM) and T lymphocyte (TL-CFU) progenitor cells after autologous and allogeneic bone marrow transplantation. Br J Haematol. 1987;65(2):137-142.

7. Yukai Lu MH, Zihao Zhang, Yan Qi, Junping Wang. The regulation of hematopoietic stem cell fate in the context of radiation. Radiation Medicine and Protection. 2020;1(1):31-34.

8. Shao L, Wang Y, Chang J, Luo Y, Meng A, Zhou D. Hematopoietic stem cell senescence and cancer therapy-induced long-term bone marrow injury. Transl Cancer Res. 2013;2(5):397-411.

9. Koukourakis MI. Radiation damage and radioprotectants: new concepts in the era of molecular medicine. Br J Radiol. 2012;85(1012): 313-330.

10. Drouet M, Mourcin F, Grenier N, et al. Single administration of stem cell factor, FLT-3 ligand, megakaryocyte growth and development factor, and interleukin-3 in combination soon after irradiation prevents nonhuman primates from myelosuppression: long-term follow-up of hematopoiesis. Blood. 2004;103(3):878-885

11. Huang P, Li X, Meng Y, et al. Interleukin-33 regulates hematopoietic stem cell regeneration after radiation injury. Stem Cell Res Ther 2019;10(1):123.

12. Zhang Y, Roos M, Himburg $\mathrm{H}$, et al. PTPsigma inhibitors promote hematopoietic stem cell regeneration. Nat Commun. 2019;10(1): 3667.

13. Velardi E, Tsai JJ, Radtke S, et al. Suppression of luteinizing hormone enhances HSC recovery after hematopoietic injury. Nat Med. 2018;24(2):239-246.

14. Mierzejewska K, Borkowska S, Suszynska E, et al. Hematopoietic stem/progenitor cells express several functional sex hormone receptors-novel evidence for a potential developmental link between hematopoiesis and primordial germ cells. Stem Cells Dev. 2015;24 (8):927-937.

15. Nakada D, Oguro H, Levi BP, et al. Oestrogen increases haematopoietic stem-cell self-renewal in females and during pregnancy. Nature. 2014;505(7484):555-558.

16. Khong DM, Dudakov JA, Hammett MV, et al. Enhanced hematopoietic stem cell function mediates immune regeneration following sex steroid blockade. Stem Cell Reports. 2015;4(3):445-458.

17. Dudakov JA, Goldberg GL, Reiseger JJ, Chidgey AP, Boyd RL. Withdrawal of sex steroids reverses age- and chemotherapy-related defects in bone marrow lymphopoiesis. J Immunol. 2009;182(10): 6247-6260.

18. Goldberg GL, Dudakov JA, Reiseger JJ, et al. Sex steroid ablation 
enhances immune reconstitution following cytotoxic antineoplastic therapy in young mice. J Immunol. 2010;184(11):6014-6024

19. Abou Dalle I, Paranal R, Zarka J, et al. Impact of luteinizing hormone suppression on hematopoietic recovery after intensive chemotherapy in patients with leukemia. Haematologica. 2021;106(4):1097-1105.

20. Poorvu PD, Barton SE, Duncan CN, et al. Use and effectiveness of gonadotropin-releasing hormone agonists for prophylactic menstrual suppression in postmenarchal women who undergo hematopoietic cell transplantation. J Pediatr Adolesc Gynecol. 2016;29(3):265-268.

21. Jadoul P, Kim SS, Committee IP. Fertility considerations in young women with hematological malignancies. J Assist Reprod Genet. 2012;29(6):479-487.

\section{BETing on rational combination therapy in mutant FLT3 acute myeloid leukemia}

Richard M. Stone

Leukemia Division, Department of Medical Oncology, Dana-Farber Cancer Institute, Boston, MA, USA

E-mail: RICHARD M. STONE - rstone@partners.org

doi:10.3324/haematol.2020.274753

A cute myeloid leukemia (AML) is a heterogenous largely intrinsically resistant bone marrow stem cell malignancy. ${ }^{1}$ While intensive therapies, including stem cell transplant, can cure some patients, these are difficult to apply and/or are ineffective in the many older patients who contract this disease. Individual patients have varying degrees of sensitivity to available agents which can be delineated based on cytogenetic and molecular disease features. About 30\% of AML patients have malignant cells whose DNA harbors a mutation in the FLT3 gene, encoding a transmembrane tyrosine kinase that transmits mitogenic signals from the extracellular space to the nucleus. ${ }^{2}$ Three-quarters of the mutations encode a duplication of from 3 to 100 amino acids in the juxtamembrane region (which is associated with an adverse prognosis); the remaining mutations are point mutations in the tyrosine kinase domain. ${ }^{2}$ Both mutations result in spontaneous dimerization and activation of the enzyme without the need for cognate ligand binding. Patients with mutant FLT3 AML are routinely treated in the upfront setting with chemotherapy plus midostaurin, a multitargeted tyrosine kinase with FLT3 inhibitory activity. ${ }^{3}$ Patients with relapsed or refractory mutant FLT3 AML can be treated with gilteritinib, a more specific and relatively well-tolerated FLT3 inhibitor, based on results of a clinical trial showing superior survival with gilteritinb compared to conventional chemotherapy. ${ }^{4}$

Unfortunately, despite the successes with midostaurin and gilteritinib in clinical trials, patients with mutant FLT3 AML frequently relapse after such therapies and are thus in need of new agents. The study of the mechanisms of resistance to FLT3 inhibitory therapy in AML is an important strategy to derive additional therapies. Patients who fail to respond or relapse after responding to gilteritinib frequently have mutations in the RAF-MAP-ERK downstream pathway. ${ }^{5}$ While there are no inhibitors of this pathway in use for leukemia, this would be one strategy to employ in combination with FLT3 inhibitors to forestall or eliminate such resistance. Levis and colleagues have suggested that bromodomain inhibition in combination with FLT3 inhibition could potentially be a useful way to overcome resistance to single-agent FLT3 inhibitory therapy (M Levis, personal observations, 2020).

Bromodomain and extra-terminal domain (BET pro- teins) are master transcriptional regulators which activate a wide variety of genes ${ }^{6}$ that are involved in cell cycle progression, leukemogenesis, and elaboration of stromal derived cytokines, the latter being important mechanisms of resistance to FLT3 inhibitors. ${ }^{7}$ FLT3 inhibitors often clear peripheral blasts but fail to eliminate bone marrow blasts, presumably due to these pro-survival cytokines. Thus, inhibition of BET proteins, including BRD 2, 3 and 4 and BRD T could be useful in preventing FLT3 inhibitor resistance. BRD 4 may be the most relevant target since it recruits an important complex involved in transcription of MYC and other genes important in promoting cell division; this complex is called the positive transcription elongation factor complex (P-TEFb).

In this edition of Haematolgica, Lee et al. show that a novel BET inhibitor, PLX51107, achieved the goal of adequate MYC suppression in humans, thereby making it an attractive agent to combine with FLT3 inhibitors. ${ }^{8}$ Could MYC downregulation with its associated decrease in cell cycle progression be useful in combination with FLT3 inhibitors such as the FLT3 ITD specific and potent agent, quizartinib?

Lee et al. make the important point that, while previous work had demonstrated synergistic cytotoxic effect of the BET inhibitor JQ1 and a FLT3 inhibitor, these experiments were performed in cell suspension culture which fails to faithfully reproduce the clinical situation. Blasts preferentially survive in the bone marrow stroma bathed in cytokines released by endothelial and other support cells. The authors of the current work showed that PXL51107 has single-agent activity against the FLT3 ITD containing human leukemia cell lines MV4-11 and MOLM14 in culture and in vivo in murine xenograft models but has no independent FLT3 inhibitory activity. This activity was synergistically increased when quizartinib was given in combination in the MV4-11 xenograft model or in primary AML cells co-cultured with bone marrow stroma. Further, plasma samples obtained from patients on a clinical trial of single-agent PLX51107 display MYC inhibition activity, suggesting that this agent possesses the requisite properties to achieve the goal of downregulation of pro-survival cytokines, making it a good candidate to combine with FLT3 inhibitors.

In summary, the preclinical work described by Lee et al. 